\title{
Random missing tooth error detection in crankshaft function of an engine control unit
}

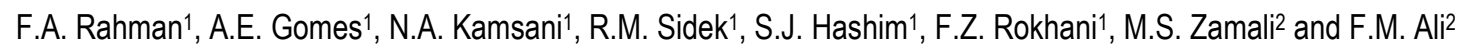 \\ ${ }^{1}$ System-on-Chip Research Group, Faculty of Engineering, Universiti Putra Malaysia, 43400 UPM Serdang, Selangor, Malaysia \\ Phone: +60397694366; Fax: +60389466327 \\ ${ }^{2}$ FSR Technology Sdn Bhd, 3, I-PARC 3 Industrial Park, Jalan Riyal U3/38, Bukit Jelutong, 40150 Shah Alam, Selangor, Malaysia
}

\begin{abstract}
Automotive industry is migrating to electronic based control which has promoted the evaluation and enhancement of engine control performance using electronic components in the research field. Modern engines are controlled by Engine Control Unit (ECU) where not only functions and control systems are in placed, but also the fail-safe mechanisms must be integrated in the ECU to ensure user safety when an error occurred. This work focuses on developing a reliable crankshaft function in an ECU using field-programmable gate array (FPGA). ABa test is carried out and the number of occurrences at unintended location are being monitored in the system to detect the random missing tooth. The reliability of the function is tested by evaluating response of the crankshaft function in an ECU when an unexpected missing tooth occurs during its operation. The developed system is able to detect the random missing tooth on a crank trigger wheel at an accuracy of $100 \%$ when the wheel is on a run at a constant wheel rotational speed. It also flags error message for further processing in other functional units of the ECU as a safe-fail mechanism for the system. Implementation of the random missing tooth detection in the crankshaft function is shown to work in the system which is developed using Verilog code.
\end{abstract}

ARTICLE HISTORY

Revised: $15^{\text {th }}$ May 2020

Accepted: $15^{\text {th }}$ May 2020

\section{KEYWORDS}

Crankshaft;

trigger wheel;

engine control unit; field programmable gate array.

\section{INTRODUCTION}

Automakers are competing and forging ahead to produce better and smarter product for consumers. This makes the operation of motor vehicles becoming more complex and automated, therefore, reducing the dependency on mechanical based control. In addition, due to the strict emission and noise regulations [1,2], electronic based controls are more favourable by the industry to cope with.

According to Denso, automotive industry started to use simple ECUs made up of analog circuits paired with fuel injection to replace carburettors in the year 1990's [3]. Gradually, more tasks and control systems which are referred as functional units are being integrated into the Engine Control Unit (ECU). Some common functional units include the Brake Control Module (BCM), General Electric Module (GEM), Powertrain Control Module (PCM) and Engine Control Module (ECM) [4, 5].

An ECU is made up of microcontrollers and it plays a vital role in a vehicle such as a high-performance motorcycle in controlling and managing the operation of the engine. Example of the operations include determining the air-to-fuel ratio, ignition timing and fuel injection based on processed input signals received from various sensors [6-8]. Some of modern ECU also controls the stability of a motorcycle $[9,10]$, anti-lock brake system (ABS) $[11,12]$ and cruise control [13].

Several works have been carried out in developing ECU prototypes for motorcycle such as in [14] where the baseline of the ECU prototype is developed using field-programmable gate array (FPGA) available in CompactRIO from National Instruments. However, the work did not focus on the fail-safe mechanism in the prototyped ECU. ECUs produced by manufacturers are limited to their own product usage and have been pre-programmed. Researchers will need to develop their own prototype in order to carry out the related investigation work. This work is carried out in collaboration with FSR Technology where the company is developing ECU for high-performance motorcycle called TUNEBOSS which is an advanced, unattached, stringless ECU for fuel injection motorcycles [15].

There are two kinds of engine for motorcycle which are the two-stroke engine and four-stroke engine. The difference between these two is that the crankshaft rotates twice to complete combustion cycle in the internal of four-stroke engine, while in two-stroke engines, crankshaft only rotates once to complete the cycle [16]. One complete combustion cycle is made up of intake, compression, power and exhaust [17]. Both engines are made up of intake port, exhaust port, piston, piston rings and crankshaft but the four-stroke engine has additional components which are camshafts, timing chains, stems, lobes, intake valves and exhaust valves.

Crankshaft drives the movement of pistons; and its position controlled the ignition timing and fuel injection timing in the engine. The timing is crucial for the engine to deliver maximum power with minimum emission in the system [18]. 
Formerly, the timing is adjusted manually on the distributor to a timing mark. Nowadays, the ignition timing and fuel injection timing is controlled by ECU using the information received from crankshaft position sensor that is normally installed at the crankshaft trigger wheel [19]. Besides that, the sensor is used to measure the rotational speed (revolution per minute, RPM) of the crankshaft.

According to Traffic Safety Facts published by National Center of Statistics and Analysis in Washington [8], the number of accidents among motorcyclist is higher than other types of vehicles' drivers. For the same distance travelled by car drivers and motorcyclist, the risk of accidents face by motorcyclist is 28 times or more [20-24]. Hence, the safety of motorcyclists is being prioritized and research towards safety and precautions of the motorcyclists are more thoroughly investigated [25, 26]. Many of the current research and development of ECU focuses not only on optimizing the control system of the engine but also on safety precautions and regaining control of the motorcycle if the rider loses control [13, 27].

Table 1 shows the comparison on recent works performed on crankshaft function in ECU. In [28], engine fault detection in car is carried out by analysing instantaneous angular acceleration of the crankshaft. From the data, fault can be observed when the instantaneous angular acceleration is abnormal. The work investigated the relationship between angular acceleration and pressure in combustion chamber. In [29], the work focuses on partitioning hardware and software of crankshaft function in an ECU of a car. No work on fault detection is carried out in [29]. The partitioning of the crankshaft function is tested using De1-SoC FPGA. Meanwhile, generation of crankshaft and camshaft signal was carried out using NXP MPC5744P microcontroller in [30]. The paper discusses on the generation of crank trigger wheel's tooth signal using NXP Model Based Design Toolbox.

Table 1. Comparison of recent works on crankshaft function in ECU.

\begin{tabular}{ccccc}
\hline Publication & Fault diagnosis & Focus & Platform & Type of engine \\
\hline $2012[28]$ & Yes & $\begin{array}{c}\text { Fault detection using crankshaft } \\
\text { instantaneous angular acceleration }\end{array}$ & dSpace & Car \\
$2017[29]$ & No & $\begin{array}{c}\text { Hardware-software partitioning of } \\
\text { crankshaft function }\end{array}$ & De1-SoC FPGA & Car \\
$2019[30]$ & No & $\begin{array}{c}\text { Generation of crankshaft and camshaft } \\
\text { signal }\end{array}$ & NXP MPC5744P & Car \\
\hline
\end{tabular}

This paper focuses on a study of unitended random missing tooth detection in a crankshaft's trigger wheel operation at a constant wheel rotational speed in ECU of a motorcycle. This work is targeted for a crankshaft's trigger wheel with 36-1 teeth application as shown in Figure 1 [31], but in this paper, result from crankshaft's trigger wheel with 10-1 teeth is being shown instead, to ease viewing of the result. The presence of the missing tooth is very important in indicating the completion of one revolution of crankshaft's trigger wheel. However, the presence of missing tooth at the unintended position may cause synchronisation issue in an ECU and thus, its proposed fail-safe mechanism is being investigated using hardware experimental setup.
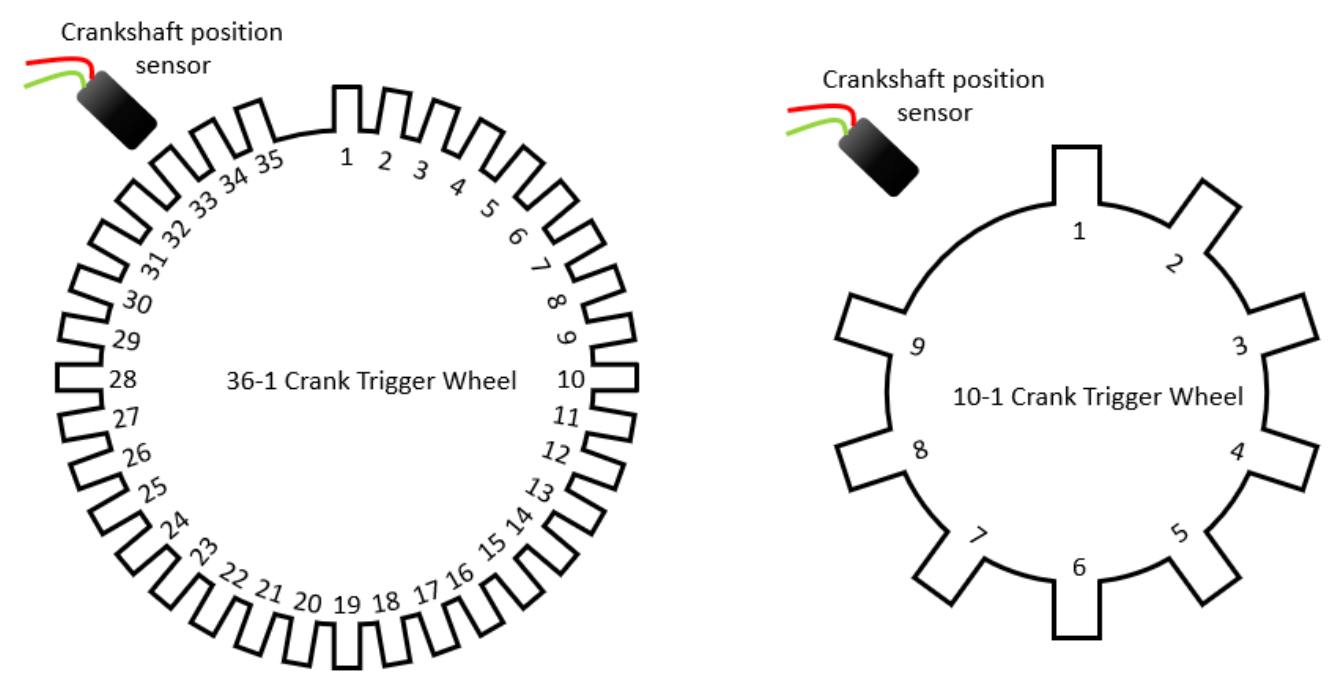

Figure 1. Illustrations of 36-1 teeth and 10-1 teeth crank trigger wheel. 


\section{FLOW OF OPERATION}

\section{General Flow of Crankshaft Function in ECU}

Figure 2 shows the general flow of crankshaft function in an ECU [32, 33]. The input signal is obtained from the reading of crankshaft position sensor. When the signal is sent to ECU, the crankshaft functional unit will determine the first and second valid edges. Gap test is performed by using the time taken from these two edges. The gap test is a test where the ECU could determine a gap (missing tooth) on the crankshaft's trigger wheel while it is running. If ECU detects no gap, the ECU will keep on running the same test on the input signal until the gap is found.

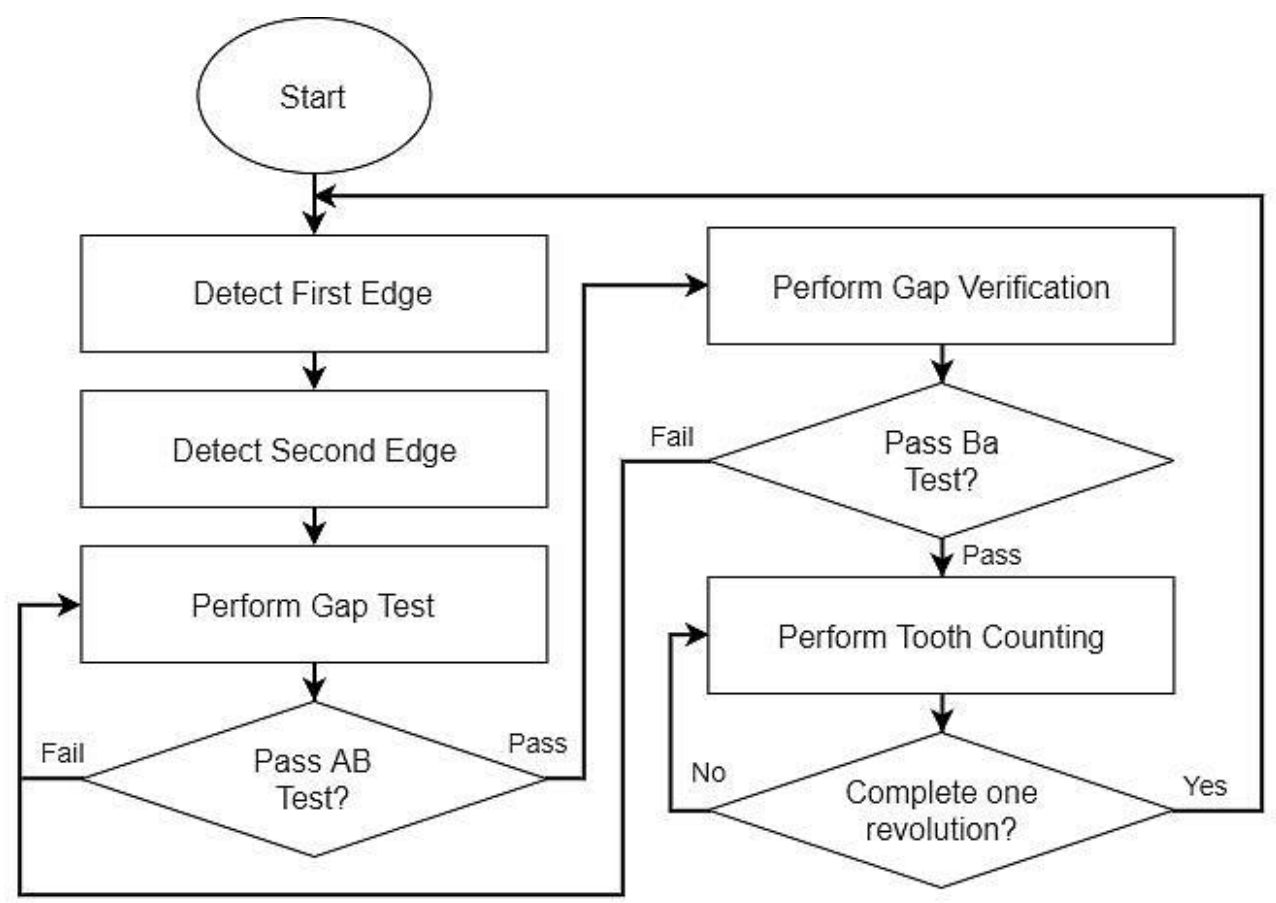

Figure 2. Flow of the crankshaft function.

There are two parts in validating the missing tooth which are gap test and gap verification. In the gap test state, $A B$ test is being carried out while in the gap verification state, $B a$ test is being performed. The period of $A, B$ and $a$ are measured as shown in Figure 3. The requirements that need to be met in order to pass this $A B a$ test are as follows:

$$
\begin{aligned}
& \text { AB test: } t_{n}>t_{n-1} \\
& \text { Ba test: } t_{n}>t_{n+1}
\end{aligned}
$$

Thus, $A B a$ test should only passed when a missing tooth is detected.

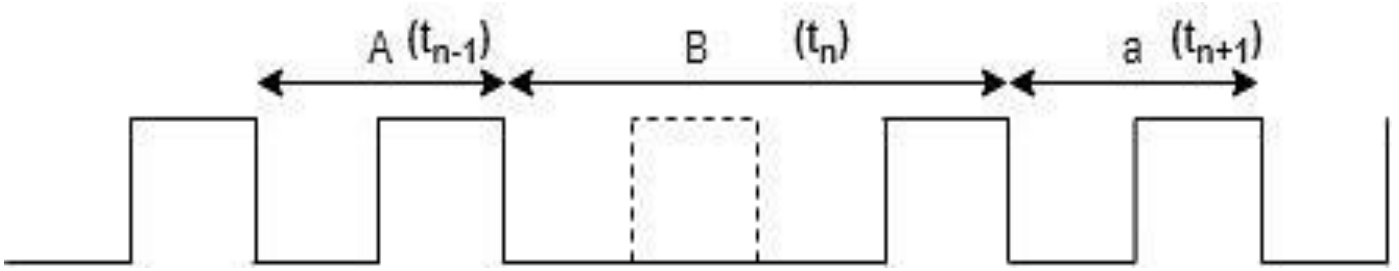

Figure 3. Example of crank trigger wheel's tooth signal.

When ECU detects the first and second edges, in this case, edges of $A$ and $B$, the period of $A$ and $B$ are stored temporarily as $t_{n-1}$ and $t_{n}$ respectively. The ECU then will perform $A B$ test where the ECU will consider the test is passed when requirements shown in Eq. (1) is met. If $A B$ test is passed, the ECU will proceed to the next state which is gap verification, also known as $B a$ test. This time, the period of $B$ and $a$ are stored temporarily as $t_{n}$ and $t_{n+l}$ respectively. The $B a$ test is considered pass if the requirement shown in Eq. (2) is met. If $A B$ test has passed but $B a$ test is failed, the system will perform the gap test and gap verification again until both $A B$ and $B a$ test are passed. Next, tooth counting state will start to count the number of teeth until one revolution is completed and then the system will go back to first edge state.

In this work, the crankshaft function shown in Figure 2 is written and compiled in Verilog code using Intel Quartus Prime software. The code is then simulated using ModelSim software to observe its functionality. Afterwards, the code 
is uploaded into DE1-SoC FPGA board. The generated input signal from the crankshaft trigger wheel's position sensor is also written in Verilog code, simulated in ModelSim software and generated in the DE1-SoC FPGA board. The input signal is assumed to behave as an ideal signal obtained from the reading of crankshaft position sensor in a real engine.

Two experiment setups based on Figure 4 are carried out where the frequencies of the generated tooth signal are varied. The developed code is executed and its output is observed through the GPIO interface on the FPGA board using an oscilloscope. In the first experiment setup, a $2.5 \mathrm{MHz}$ input signal taken from crankshaft position sensor is generated using FPGA. Meanwhile in the second experiment setup, input signal with frequency of $30 \mathrm{kHz}$ is being generated using FPGA and signal generator. By varying the frequency of the input clock signals, the frequency of the tooth generated also varied. This experiment is performed in order to validate the code with varying frequencies of square waveforms with and without the GPIO interface.

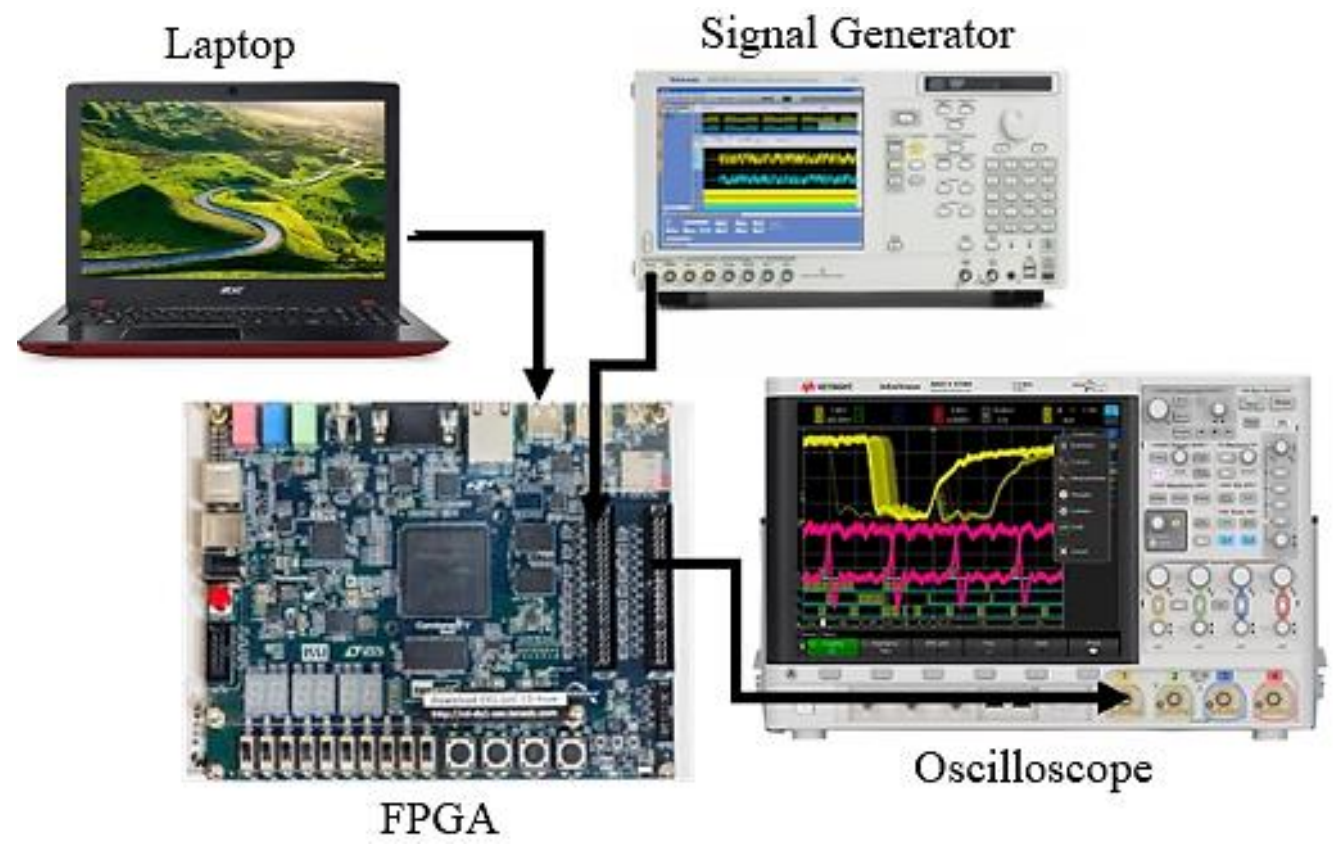

Figure 4. Experimental setup.

\section{Random Missing Tooth}

The crank trigger wheel is subjected to wear and tear issue because of rotating movement in the engine [34]. Some of the crank trigger wheel's tooth might be worn out (shape degradation) due to mechanical friction and harsh environment present around the engine. In addition to that, metal residue from parts in the engine could also filling in the gap between the teeth and cause misreading of the magnetic based sensor. There are also cases where the crank trigger wheel's tooth is broken thus causing unwanted missing tooth presence detection. The random missing tooth occurrence will result in synchronization issue and can lead to incorrect decision made by the ECU. These situations can cause safety issue to the user where in the worst case scenario, the engine might explode due to incorrect timings for the engine to properly function.

Due to the cases discussed above, the signal obtained from the crankshaft position sensor will contain wrong information about the crank trigger wheel and hence introduce synchronization issue in the engine. Thus, a reliable crankshaft function must be developed in order to identify and distinguish the unintended random missing tooth occurrence in the ECU. The proposed flow for the missing tooth detection is shown in Figure 5.

In the proposed flow, $A B a$ test is still being performed by the ECU upon receiving the input signal from crank trigger wheel position sensor. This test will determine the period of each pulse signal produced by the sensor and processed its information. In general, if a missing tooth is detected by the ECU, this missing tooth will pass the $A B a$ test. However, if the $A B a$ test has passed and still a missing tooth is detected in any state other than the expected counting state, the system will reset its counter, set an error flag and restart counting to the first edge state.

In the case of a missing tooth being detected after it has passed the $A B a$ test and it is still in the counting state (which means it is not at the expected location), the ECU will ignore the first time error. The ECU will continue to count the number of teeth and compensate the number of teeth counted in the next tooth count. For example, if the ECU is counting until $7^{\text {th }}$ tooth and there is a random tooth missing at $8^{\text {th }}$ tooth location, the tooth count will continue to 9 at the next tooth detected. But if there is a second time missing tooth occurrence at the unexpected location after that, the system will regard this as an error and it will restart the cycle back to first edge state.

The justification of ignoring the first error in the function is that the sensor might misread the tooth, or occurrence of impurities that is blocking in between the teeth temporarily that leads to sensor misread. If the error occurred again for the second time or more, it is confirmed that the missing tooth is present at that specific tooth location. In the case of 
permanent blockage between the tooth gap, the system will consider the tooth to be missing as the sensor cannot detect the edge of the tooth. When the unexpected missing tooth presence is confirmed, the crankshaft function will send the error flag to the ECU for further action. Actions that can be taken by the ECU are to stop the engine immediately or to light up check engine indicator.

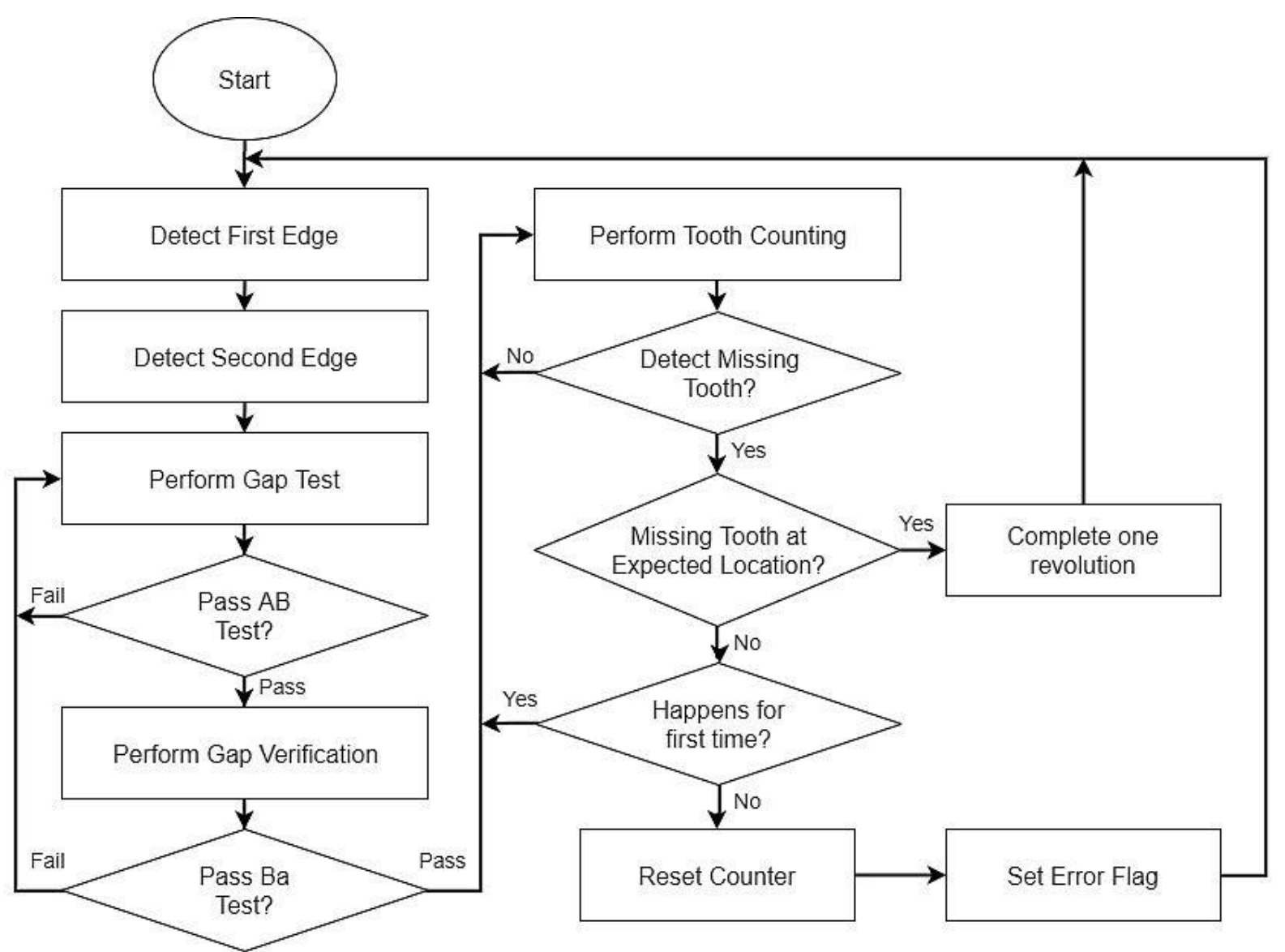

Figure 5. Proposed flowchart of missing tooth detection added into the crankshaft function.

In order to observe the functionality of the crankshaft function during a random missing tooth, DE1-SoC FPGA board is used as before but a random missing tooth generation code is added to the crankshaft function. The random missing tooth is set to occur when a switch on the FPGA board is turned on. For viewing simplicity, a 10-1 teeth signal is used instead of the 36-1 teeth signal. Simulation is first being carried out using ModelSim software to check for the functionality of the developed code. The function of the code is then being verified using the same hardware setup as shown in Figure 4. A switch on the DE1-SoC FPGA board is set to be used as the trigger switch for the missing tooth to happen. The switch is turned on manually by the user to indicate the unexpected missing tooth occurrence in the signal.

\section{RESULTS AND DISCUSSIONS}

To ease observation, crankshaft function of 10-1 teeth crank trigger wheel is simulated. The code is shown to work at both frequencies, $2.5 \mathrm{MHz}$ and $30 \mathrm{kHz}$ as shown in Figure 6, Figure 7 and Figure 8 . The frequency of the signal is related to the rotational speed of the crank trigger wheel where it could vary in the system. Hence the code must be validated across a certain frequency range and it should be able to detect the missing tooth accurately. However in this work, the frequency is assumed to be at a constant wheel rotational speed during the validation. The chosen frequencies, $2.5 \mathrm{MHz}$ is the maximum frequency of input signal calculated when using FPGA's internal clock to generate the input signal and $30 \mathrm{kHz}$ which is the maximum frequency of input signal calculated when generating input signal using the signal generator. These frequencies are chosen because it is the highest frequency that could be achieved in the harware settings, limited by hardware capabilities. Figure 6(a) shows the tooth generated signal using FPGA while Figure 6(b) shows the tooth signal generated using FPGA and signal generator. It can be observed that the code is able to generate 10-1 teeth of crank trigger wheel signal with varied frequencies. The signals shown in Figure 6 are generated to behave like the signal obtained from the crank shaft position sensor, which is used as an input to the crankshaft function in the ECU. Figure 7 and Figure 8 shows the result of developed code with gap test function using the first experiment setup (FPGA only). 


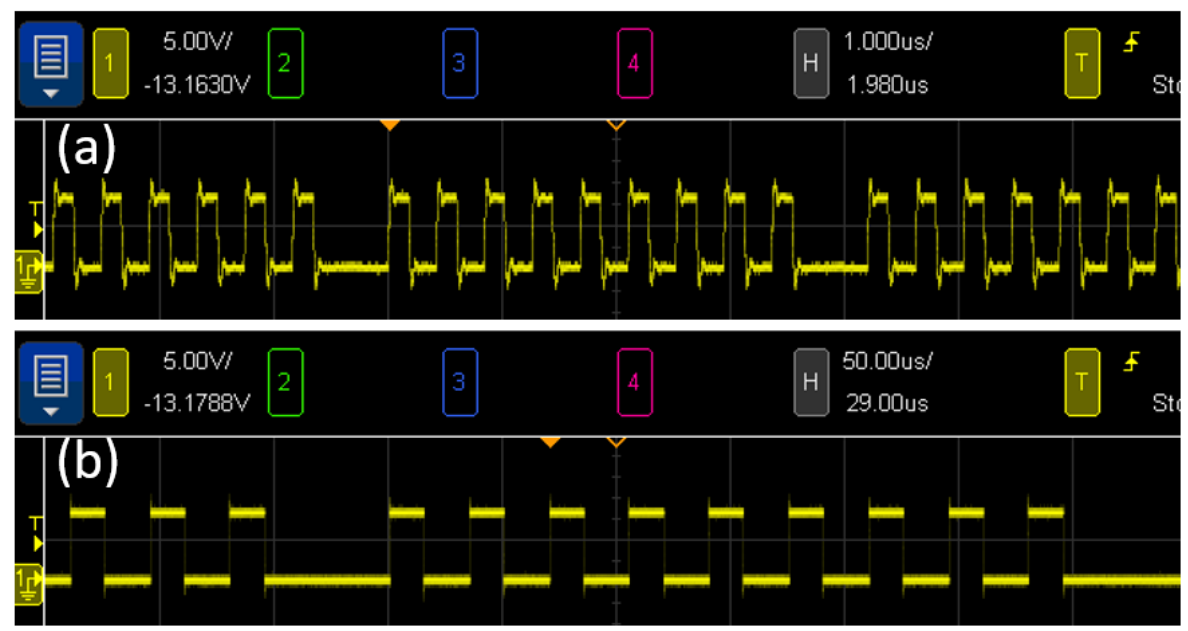

Figure 6. Output observed for 10-1 tooth generation using (a) FPGA and (b) FPGA and signal generator.

Figure 7 shows the waveform of the output signal of 36-1 crank trigger wheel position sensor, $A B$ test and $B a$ test. The frequency of one complete revolution of crankshaft simulated using FPGA board is $69.4 \mathrm{kHz}$. Similar work could be observed in [35] where 36-1 crank trigger wheel's tooth has been generated at frequency of $166.7 \mathrm{~Hz}$ using LabView targeting for four-stroke engine application. In Figure 7, the $A B$ test which is also known as gap test shows high output after the $A B$ test state is done and the test is passed. When the result at $A B$ test shows low output, it means that $A B$ test is failed, and no missing tooth gap is detected. Similar sequence is also expected for $B a$ test. The code is shown to work at varied frequency and with different number of teeth on the crank tringger wheel.

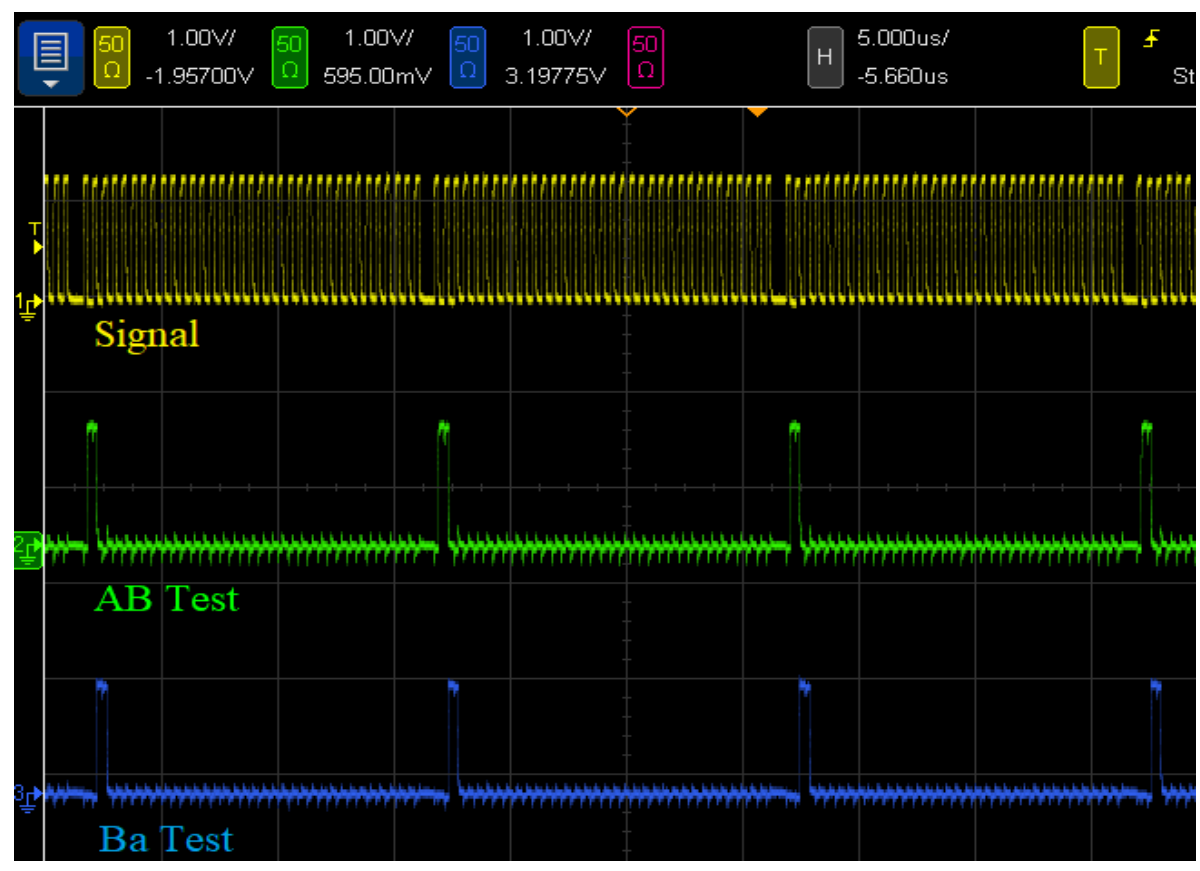

Figure 7. Signal of 36-1 crank trigger wheel with verified $A B a$ gap test.

Figure 8 shows a clearer observation on the process of gap testing and gap verifying. For every complete cycle of tooth detected, new $t_{n}$ values are recorded. $A B$ test and $B a$ test are checked with the requirements shown in Eqs. (1) and (2) but the result only shows high output when the requirements are fulfilled which means that there is a missing tooth shown in the crank wheel tooth signal. 


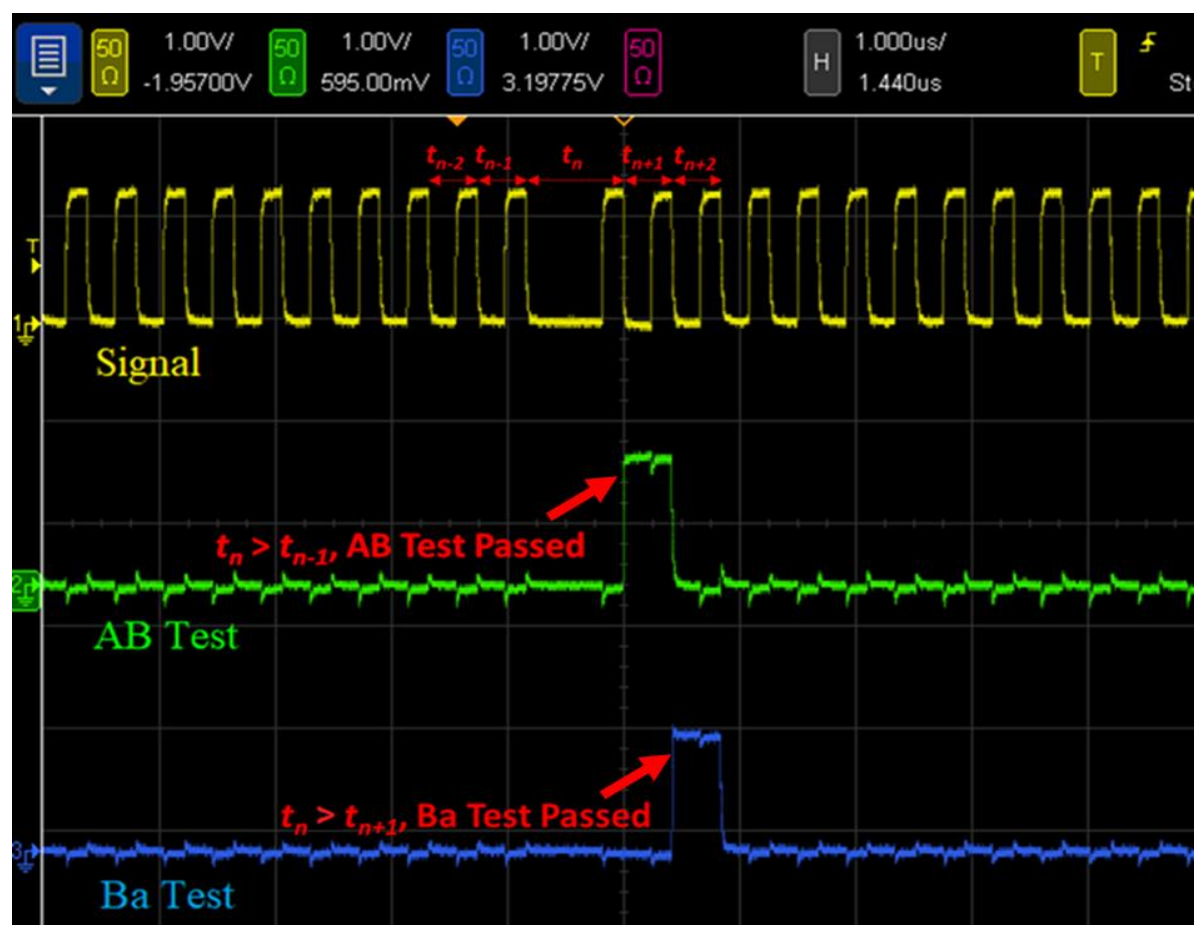

Figure 8. Clearer view showing that the signal passed the $A B$ test shown in green while $B a$ test is shown in blue.

\section{Random Missing Tooth}

Figure 9 shows the simulation of 10-1 teeth of crank trigger wheel in ModelSim software with the occurrence of random missing teeth. $C L O C K_{-} 50$ refers to the internal clock that is being used to generate the crank trigger wheel tooth signal, $S W$ refers to the switch that is used to trigger the random missing tooth occurrence and signal shows the generated crank trigger wheel tooth signal. ABtest and Batest show the results of $A B$ and $B a$ tests at every detection of missing tooth occurrence and the last waveform indicates the unexpected missing tooth error where the error waveform will go high when an error is detected.

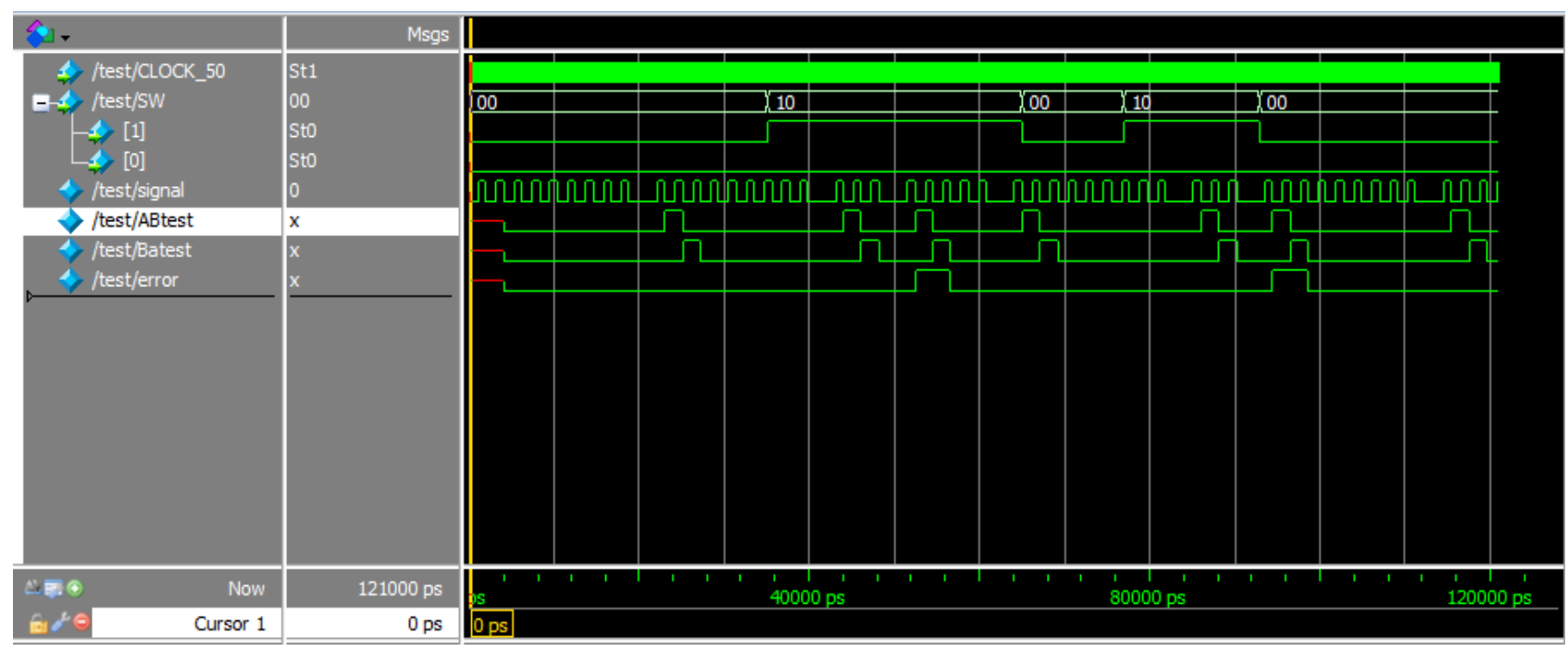

Figure 9. Simulation of 10-1 crank trigger wheel done in ModelSim.

As seen in Figure 9, the $A B$ test and $B a$ test identifies any missing tooth that occur from the signal, but the error is only triggered if the second random missing tooth occurs. Once the first random missing tooth occurs at the $4^{\text {th }}$ tooth and ECU detects the error, the counter continues to count the tooth, compensate the missing tooth and continues to count the tooth so the next tooth detected is the $5^{\text {th }}$ tooth as shown in Figure 9 and Figure 10. However, when the second random missing tooth is detected, the system will indicate this event as a random missing tooth error and reset the counter to zero as shown in Figure 11. 

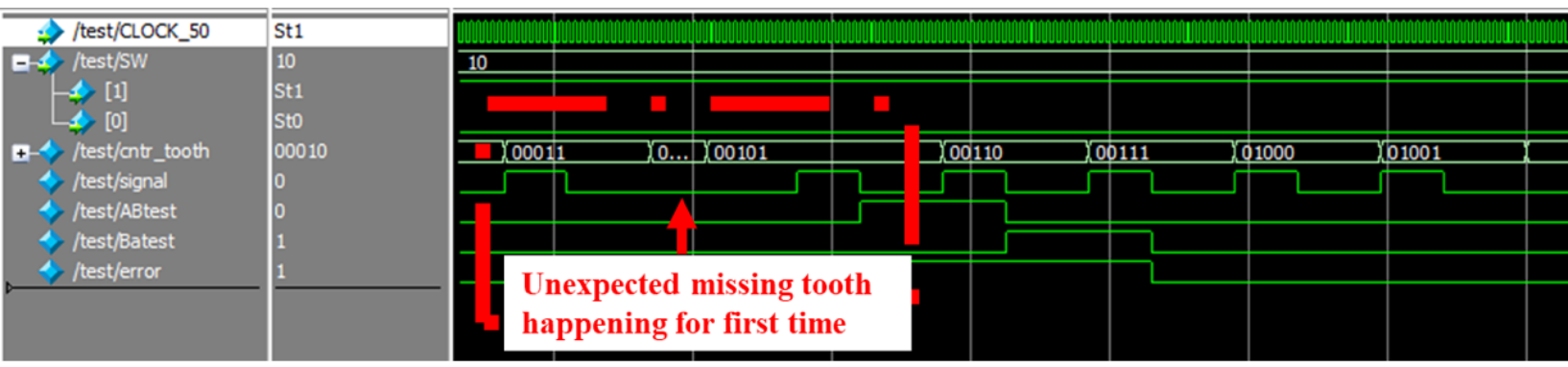

Figure 10. Unexpected missing tooth occur at $4^{\text {th }}$ tooth for the first time so the counter continues to count and compensate the missing tooth so the next signal detected is counted as $5^{\text {th }}$ tooth.
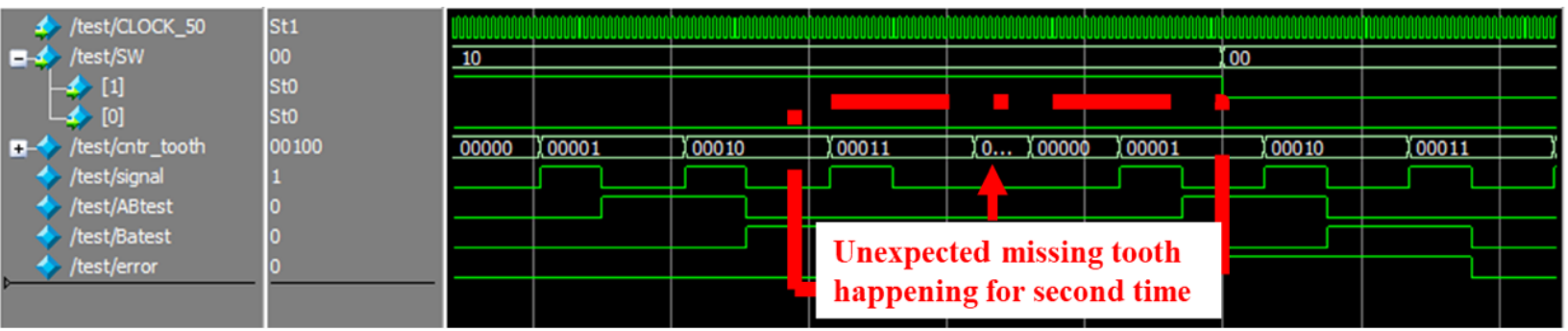

Figure 11. Unexpected missing tooth occur at $4^{\text {th }}$ tooth for the second time. The system will treat the event as a random missing tooth error and hence reset the counter to zero.

The simulation shown in Figure 9 shows the effect of the unexpected missing tooth error that occurs in ModelSim. Figure 12 and Figure 13 shows the result of detection of random missing teeth of the crank trigger wheel signal observed via an oscilloscope. Figure 12 shows 10-1 teeth of crank trigger wheel signal when no random missing teeth occurs (in this case, the $S W$ switch that is set to trigger the random missing tooth is not triggered). The gap test is executed, and the missing tooth is identified after one complete revolution. $A B$ test and $B a$ test are performed every time the ECU detects the tooth gap and when the missing tooth occur at the expected location, the results will show that the $A B a$ test is passed and the error signal is not flagged.

Figure 13 shows the 10-1 crank trigger wheel signal when the $S W$ switch is triggered. When the $S W$ switch is ON, the signal will keep on having an unexpected missing tooth (in this case it occurred at every fourth teeth). When the system identifies the missing tooth, $A B$ test and $B a$ test are performed. As the requirements for missing gap is fulfilled, both $A B$ and $B a$ tests are passed but the error signal is flagged (goes high) indicating that this is an error and missing tooth is unexpected at that state. Note that in Figure 13 also, the error occurs at the $4^{\text {th }}$ tooth is not the error that occurs for the first time. Hence, the tooth count resets and starts from 1. Therefore, any subsequent random missing tooth that occurs after one random missing tooth, will act as a reset for the counter and the signal.

Error occurrence in engine can be classified as fatal or non-fatal error. A fatal error will cause the engine to stop immediately before a dangerous consequence such as engine explosion could happen. A non-fatal error will not cause the engine to stop immediately but the ECU will send signals to other functions such as check engine light of which it will be lighten up to alert the user on the engine condition. In this work, an error signal is generated after a random missing tooth error is detected in the system and it can be processed by the ECU to warn the motorcyclist or stop the engine depending on the classified error in the ECU.

Table 2 shows the comparison on recent works performed on crank trigger wheel's tooth generation. The works generate the same configuration of crank trigger wheel's tooth with different number of teeth. The only difference is that both in [35] and [30] shows no study on the reliability of the tooth generated because the papers focused on the synchronization between crankshaft and camshaft rotation. However, this paper focuses on the reliability of the missing tooth detection where gap test and gap verification are being used to ensure that the generated tooth and the missing tooth occurrence are as intended. Even though the board used in [30] is smaller in size in comparison to De1-SoC, De1-SoC could handle Verilog language where in future work, hardware implementation of the crankshaft function can be carried out and it could increase the processing speed of the ECU as the function could be partitioned between software and hardware as studied in [29]. 


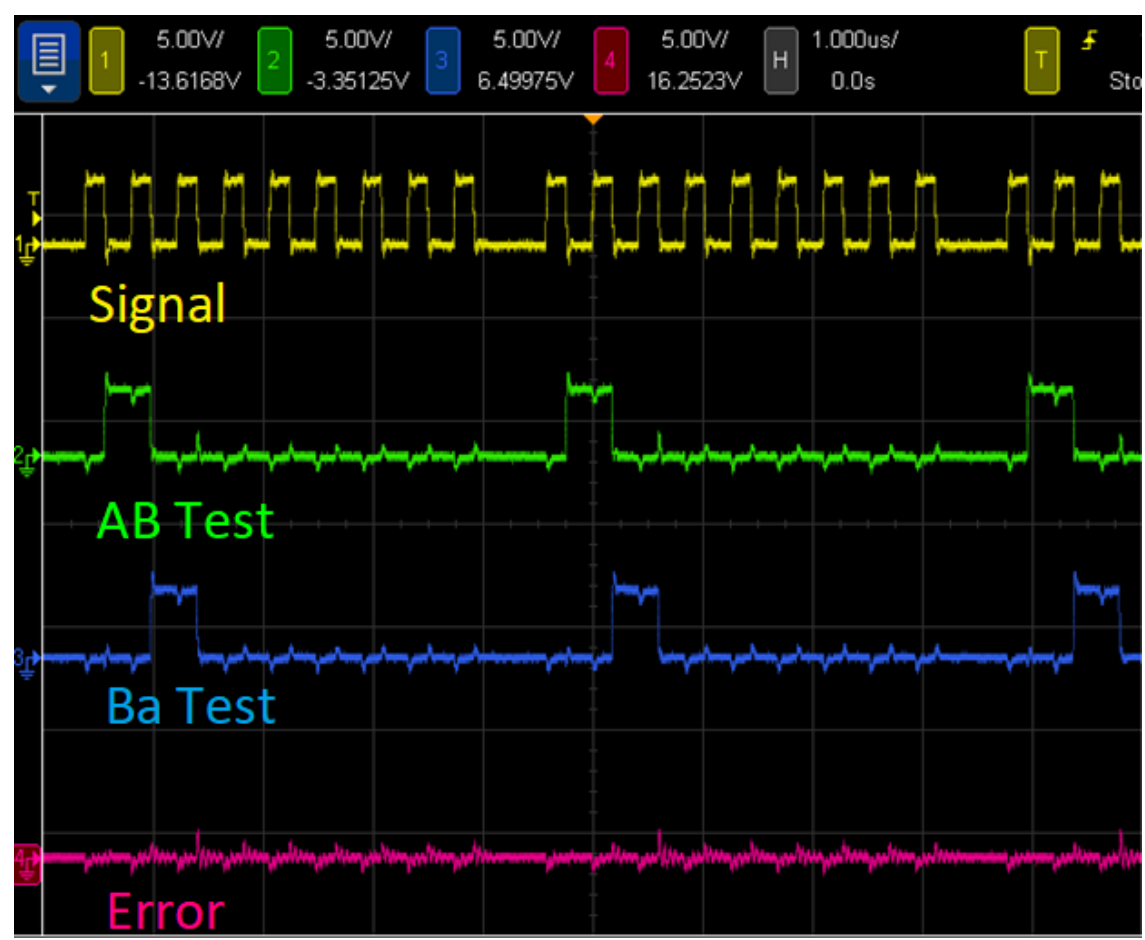

Figure 12. Signal of crank 10-1 crank trigger wheel observed from oscilloscope with no random missing tooth error.

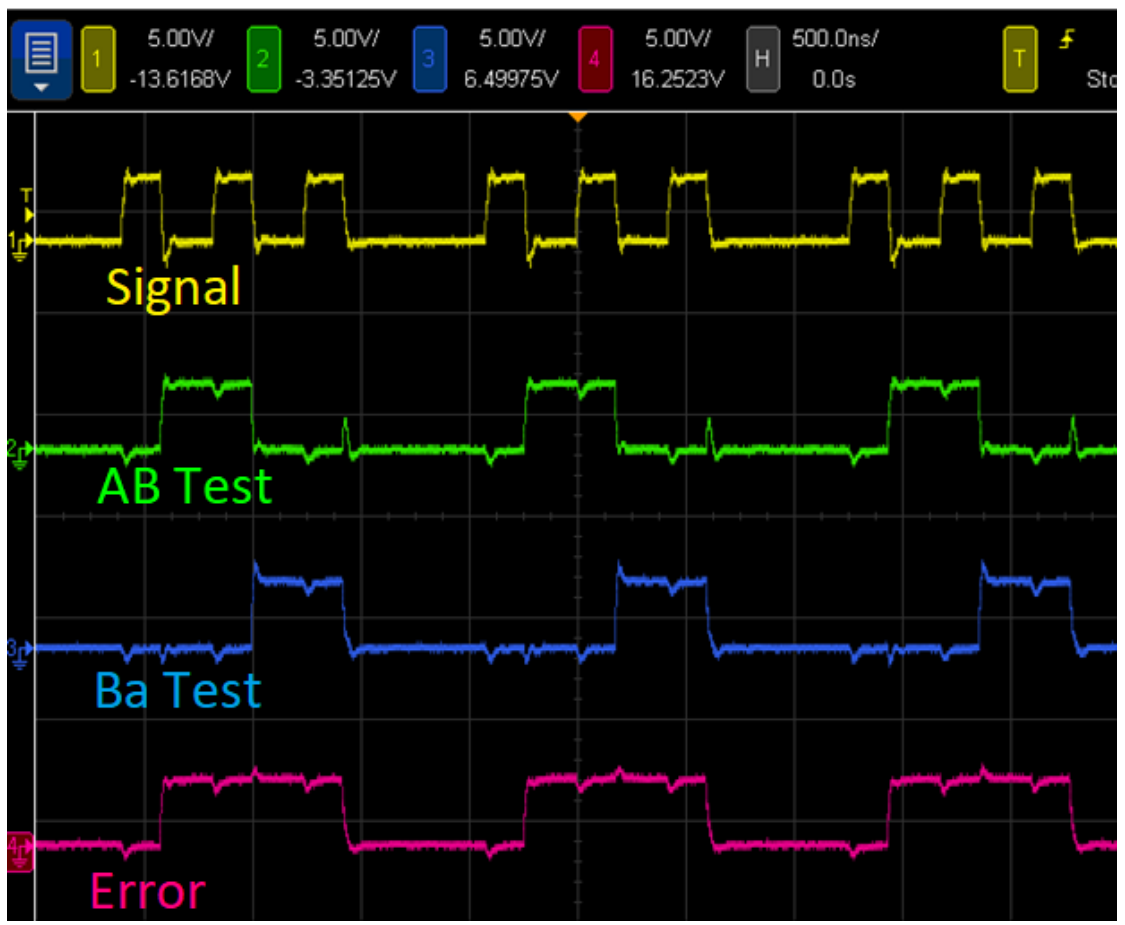

Figure 13. Signal of 10-1 crank trigger wheel observed from oscilloscope with random missing tooth error. 
Table 2. Comparison of recent works on crank trigger wheel's tooth generation.

\begin{tabular}{ccccccc}
\hline Publication & $\begin{array}{c}\text { No. of tooth } \\
\text { generated }\end{array}$ & Speed & RPM & Software & Hardware & $\begin{array}{c}\text { Study on tooth } \\
\text { generation } \\
\text { reliability }\end{array}$ \\
\hline $2017[35]$ & $36-1$ and 60-2 & $\begin{array}{c}166.67 \mathrm{~Hz} \\
\text { (Both) }\end{array}$ & 10000 & $\begin{array}{c}\text { LabView and } \\
\text { Veristand } \\
\text { Simulink and NXP } \\
\text { Model Based } \\
\text { Design Toolbox }\end{array}$ & $\begin{array}{c}\text { MPC5744P } \\
\text { microcontroller }\end{array}$ & NI-9401 cRIO \\
This work & $60-2$ & $16.67 \mathrm{~Hz}$ & 1000 & $\begin{array}{c}\text { No } \\
\text { Intel Quartus Prime }\end{array}$ & De1-SoC FPGA & Yes \\
\hline
\end{tabular}

\section{CONCLUSIONS AND FUTURE WORK}

The operation of the crankshaft function in engine control unit (ECU) targeted for motorcycle application is successfully developed, tested and verified across a range of frequencies that is related to the wheel rotational speed during its use. The crankshaft function is implemented in Verilog language; and its functionality is observed in Modelsim and also on DE1-SoC FPGA board. Proposed solution in detecting an unexpected random missing tooth has been integrated into the crankshaft function and it has shown to be able to detect the error in the signal with $100 \%$ accuracy at a constant wheel rotational speed. By integrating the detection scheme, the reliability of the operation of the crankshaft function in ECU is enhanced. The system will continue to count the tooth when an error occurred for the first time but will reset the counter if the error happens twice. An error signal that indicates the error detection is generated for this function and could be used as an input to other functions in the ECU to implement a fail-safe mechanism for the engine.

In future work, crankshaft function running in acceleration and deceleration modes and noise filtering in input signal will be included for improvement in the crankshaft function. Additionally, an interface for end user is needed so that the users can know real-time status of the engine.

\section{ACKNOWLEDGMENTS}

The author thanks Collaborative Research in Engineering, Science and Technology (CREST) grant, P28C1-15 for the support.

\section{REFERENCES}

[1] R. Lewis, R. Zako, A. Biddle, and R. Isbell, "Reducing Greenhouse Gas Emissions from Transportation and Land Use: Lessons from West Coast States," The Journal of Transport and Land Use, vol. 11, no. 1, pp. 343-366, 2018.

[2] M. S. Pshtiwan, H. A. Aziz, A. As'arry, K. A. M. Rezali, M. M. Noor, M. Norhafana, M. A. A. Mossa, and S. M. Shareef, "International Regulation of Vehicle Emissions Control Rules and Its Influence on Academic Engine Development Experimental Study and Vehicle Manufacturing," presented at the $1^{\text {st }}$ Internal Postgraduate Conference on Mechanical Engineering (IPCME2018), 2018.

[3] A History of Engine Management Systems according to DENSO, DENSO, 01 Feb. 2019. [Online]. Available: https://www.denso-am.eu/media/corporate-news/2017/march-2017-newsletter-a-history-of-engine-management-systemsaccording-to-denso/

[4] A. Malik, S. W. Shah, and A. J. Khan, "Design of Vehicle Health and Position Telemetry System for Management," International Journal of Scientific \& Engineering Research, vol. 9, no. 4, pp. 1248-1254, 2018.

[5] B. Ashok, A. Denis, and K. Ramesh, "A Review on Control System Architecture of a SI Engine Management System," Annual Reviews in Control, vol. 41, pp. 94-118, 2016.

[6] T. Wen-Chang, W. Zong-Hua, Y. Peng-Cheng, and C. Shyue-bin, "Design of Electrical Driving Circuits for a High-pressure Fuel Injector and Control of Fuel Injection Quantities by using the Polynomial Curve Fitting Method," International Journal of Intelligent Information Processing, vol. 2, no. 2, pp. 60-73, 2011, doi: 10.4256/ijiip.vol2.issue2.7.

[7] C. Pascal, D. A. Camillo, and C. Massimo, "Traction Control System for Motorcycles," EURASIP Journal on Embedded System, 2009, doi: 10.1155/2009/161373.

[8] A. Kamarudin, A. Riza, and I. Rozmi, "Intelligent Transport System for Motorcycle Safety and Issues," European Journal of Scientific Research, vol. 28, no. 4, pp. 600-611, 2009.

[9] D. F. Pierpaolo, T. Mara, and M. S. Sergio, "Electronic Stability Control for Powered Two-Wheelers," presented at the IEEE Transactions on Control Systems Technology, 2014.

[10] P. Kalaiselvan, K. Ramarethinam, and P. Pandiaraj, "Design of Stability Control Motorbike with Abs and Crash Location Sensing," International Journal of Science and Research (IJSR), vol. 3, no. 3, pp. 281-285, 2014. 
[11] H. Chun-Kuei and S. Ming-Chang, "Design of a Hydraulic Anti-Lock Braking System (ABS) for a Motorcycle," Journal of Mechanical Science and Technology, vol. 24, no. 5, pp. 1141-1149, 2010, doi: 10.1007/s12206-010-0320-9.

[12] A. I. Shaikh and B. S. Brij, "Modelling and Comparative Analysis of Anti-Lock Braking System (ABS) Control Strategies for Motorcycle Based on MATLAB/Simulink," International Journal for Scientific Research \& Development (IJSRD), vol. 7, no. 1427-1430, 2019.

[13] C. Thiagarajan and T. Muthumanikam, "Autonomous ECU Monitored Automatic Cruise Control System for Bikes and Motorcycles," International Journal of Advanced Research in Management, Architecture, Technology and Engineerning (IJARMATE), vol. 3, no. 12, pp. 9-12, 2017.

[14] C. Dase, J. S. Falcon, and B. Maccleery. (2006) Motorcycle Control Prototyping using an FPGA-based Embedded Control System. IEEE Control Systems Magazine. 17-21.

[15] TuneBoss - High Performance ECU by FSR Technology, 25 Dec. 2019. [Online]. Available: https://tuneboss.com/

[16] R. J. Ragnarsson, "Engine Control Unit for a 4 Cylinder Motorcycle Engine," Bachelor, School of Science and Engineering, University of Reykjavik, 2017.

[17] M. Gustafsson, "Crank Angle Based Virtual Cylinder Pressure Sensor in Heavy-Duty Engine Application," Master, Department of Electrical Engineering, Linkoping University, 2015.

[18] K. Tasaki, "Research on Misfire Detection Algorithms for Motorcycle Engine Firing at Uneven Intervals," presented at the Keihin Technical Review, 2018.

[19] P. G. Manoj and R. T. Patil, "Electronics Control Unit (ECU) for Dual Fuel HCCI Engine," International Journal of Recent Trends in Engineering \& Research, vol. 2, no. 6, pp. 470-474, 2016.

[20] H. R. More, A. A. Digrase, and A. V. Wayse, "Linear PID Control Technique for Single Wheel ABS (Anti-lock Braking System) of Motorcycle," presented at the 2017 2nd International Conference for Convergence in Technology (I2CT), 2017.

[21] F. Z. Rokhani, M. T. A. Rahman, N. A. Kamsani, M. I. Saripan, K. Samsudin, and M. K. Hassan, "ASIL Determination for Motorbike's Electronic Throttle Control System (ETCS) Mulfunction," European Physical Journal-Web Conference, vol. 162 , pp. 1-5, Aug 20172017.

[22] National Center for Statistics and Analysis, "Motorcycles | 2016 Data," in "Traffic Safety Facts," Washington, DC, DOT HS 812 492, 2018.

[23] L. R. Megan, J. M. Michael, B. B. Janette, R. C. Peter, and A. C. Melissa, "Correlates of Motorcycle Helmet Use Among Recent Graduates of a Motorcycle Training Course," Accident Analysis and Prevention, vol. 42, no. 6, pp. 2057-2962, November 2016

[24] International Traffic Safety Data and Analysis Group (IRTAD), "Road Safety Annual Report 2019," France, 2019.

[25] M. T. Muhammad Fauzi, N. M. Muhammad Izzat, R. Helmi, A. Ismail Nasiruddin, W. F. Wan Muhammad Syahmi, O. Abdul Rahman, and J. Roseleena, "The Explorations in Defining Motorcycling Fatigue: a Pilot Study," Bioengineering and Ergonomics, vol. 76, no. 7, pp. 115-118, 2015.

[26] S. Mohd Syazwan, H. Aqbal, H. Azhar, M. Mohd Hafzi, M. Zulhaidi, M. Nur Izzi, and H. Mohd Rosli, "Exploratory Study on Airbag Suitability for Low Engine Capacity Motorcycles," Jurnal Teknologi (Sciences and Engineering), vol. 78, no. 4, pp. 65-69, 2016.

[27] G. Rupesh, G. Sheifali, G. Bhavneet, K. Dhruv, R. Chandan, and G. Ishaant, "Improved Mechanism for Converting Gear Transmission of Motorcycle from Manual to Automatic," Indian Journal of Science and Technology, vol. 9, no. 48, 2016.

[28] L. K. Binh and J. Tuma, "Approach to Gasoline Engine Faults Diagnosis Based on Crankshaft Instantaneous Angular Acceleration," presented at the 2012 13th International Carpathian Control Conference (ICCC), 2012.

[29] Y. Khai Chein, R. Mohd Fariq, A. R. Fatimah, K. Noor Ain, and R. Fakhrul Zaman, "Hardware Software Partitioning of Crankshaft Function in Engine Control Units using FPGA-based Testing," presented at the 2017 IEEE 15th Student Conference on Research and Development (SCORED), 2017.

[30] S. Gulgonul and N. Sozbir, "Crank Cam Signal Generator using NXP Model Based Design Toolbox," presented at the 7th International Symposium on Innovative Technologies in Engineering and Science, Turkey, 2019.

[31] EFI 36-1 Trigger Wheel, 3 Feb. 2019. [Online]. Available: http://arengineering.com/products/efi-36-1-trigger-wheel/

[32] G. Emerson and E. Kilbride, "Using the Engine Position (CRANK and CAM) eTPU Functions," ed: NXP Semiconductors, 2009.

[33] B. Milan, "Engine Control eTPU Library," AN4907, 2016.

[34] G. Ramesh, I. Narasimharao, and A. Robinson, "Intelligent Engine with Micro Controller Valve Actuation and Eliminating the Cam Linkage Arrangement," presented at the Frontiers in Automobile and Mechanical Engineering (FAME), 2010.

[35] R. Drosescu, "Virtual Engine Management Simulator for Educational Purposes," presented at the CAR2017 International Congress of Automotive and Transport Engineering, 2017. 